Rechtsfolgen des

Refinanzierungsregisters 
Khanh Dang Ngo

\section{Rechtsfolgen des \\ Refinanzierungsregisters}

Die gesetzlichen Wirkungen der Eintragung nach $\S 22 \mathrm{j}$ KWG

Springer Gabler 
Khanh Dang Ngo

Frankfurt am Main, Deutschland

D 6

Zugl.: Münster (Westf.), Univ., Diss. der Rechtswissenschaftlichen Fakultät, 2012

ISBN 978-3-658-00478-1

ISBN 978-3-658-00479-8 (eBook)

DOI $10.1007 / 978-3-658-00479-8$

Die Deutsche Nationalbibliothek verzeichnet diese Publikation in der Deutschen Nationalbibliografie; detaillierte bibliografische Daten sind im Internet über http://dnb.d-nb.de abrufbar.

Springer Gabler

(C) Springer Fachmedien Wiesbaden 2013

Das Werk einschließlich aller seiner Teile ist urheberrechtlich geschützt. Jede Verwertung, die nicht ausdrücklich vom Urheberrechtsgesetz zugelassen ist, bedarf der vorherigen $\mathrm{Zu}$ stimmung des Verlags. Das gilt insbesondere für Vervielfältigungen, Bearbeitungen, Übersetzungen, Mikroverfilmungen und die Einspeicherung und Verarbeitung in elektronischen Systemen.

Die Wiedergabe von Gebrauchsnamen, Handelsnamen, Warenbezeichnungen usw. in diesem Werk berechtigt auch ohne besondere Kennzeichnung nicht zu der Annahme, dass solche Namen im Sinne der Warenzeichen- und Markenschutz-Gesetzgebung als frei zu betrachten wären und daher von jedermann benutzt werden dürften.

Gedruckt auf säurefreiem und chlorfrei gebleichtem Papier

Springer Gabler ist eine Marke von Springer DE. Springer DE ist Teil der Fachverlagsgruppe Springer Science+Business Media.

www.springer-gabler.de 
Meinen Eltern 


\section{Vorwort}

Die vorliegende Arbeit wurde von der Rechtswissenschaftlichen Fakultät der Westfälischen Wilhelms-Universität Münster im Sommersemester 2012 als Dissertation angenommen.

In erster Linie bedanken möchte ich mich bei meinem Doktorvater, Herrn Prof. Dr. Matthias Casper, der diese Untersuchung angeregt und die Arbeit kontinuierlich und mit großem Engagement betreut hat. Mit seinen Anregungen und seiner Unterstützung trug er wesentlich zum Gelingen der Arbeit bei. Herrn Prof. Dr. Ingo Saenger möchte ich für die zügige und wohlwollende Erstellung des Zweitgutachtens herzlich danken.

$\mathrm{Zu}$ großem Dank bin ich auch Frau Prof. Tamar Frankel verpflichtet. Sie hat mir nicht nur ein Verständnis für das US-amerikanische trust law, sondern vor allem die wiederentdeckte Freude an der kritischen Betrachtung von rechtlichen Grundsätzen vermittelt.

Bedanken möchte ich mich recht herzlich bei allen Freunden und Kollegen, die die Durchführung meiner Arbeit stets ideenreich und tatkräftig begleitet haben. Sie alle an dieser Stelle separat zu benennen, wird nicht möglich sein.

Ganz herzlich möchte ich mich bei Frau Leonie Maria Odilia Kremer bedanken, die die Fertigstellung dieser Arbeit leider nicht mehr miterleben konnte. Sie hat mir jedoch den Willen, stets bis ans Ende zu gehen, mit auf den Weg gegeben und wird mir im ständigen Gedenken verbunden bleiben.

Mein größter Dank gilt meinen Eltern, Frau Thi Phuong Huynh und Herrn Ngoc Thanh Ngo, für ihre liebevolle Unterstützung über die vielen Jahre. Für mich sind sie stets die umsichtigsten Berater und größten Vorbilder, die sich ein Sohn wünschen kann. Ihnen sei diese Arbeit gewidmet.

Frankfurt a.M.

Khanh Dang Ngo 


\section{Inhaltsübersicht}

Vorwort VII

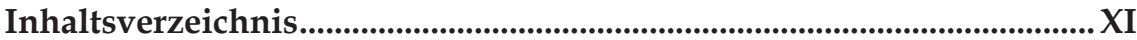

\section{Kapitel 1: Einführung}

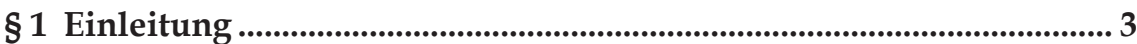

I. Gegenstand der Untersuchung....................................................... 3

II. Anlass der Untersuchung................................................................ 5

III. Themeneingrenzung und Gang der Untersuchung ........................ 7

§ 2 Entstehungsgeschichte der Vorschriften §§ 22a-22o KWG................ 9

I. Gesetzgeberische Bemühungen zur Förderung des

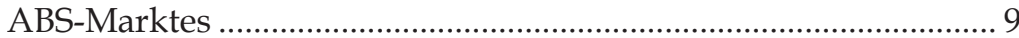

II. Politischer Vorprozess zur Schaffung eines

Verbriefungsregisters........................................................................ 19

III. Referentenentwurf zur Schaffung des

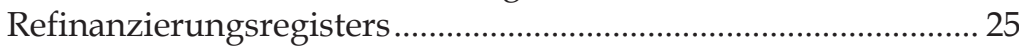

IV. Gesetz zur Schaffung eines Refinanzierungsregisters................... 28

V. Gesetz zur Fortentwicklung des Pfandbriefrechts.......................... 34

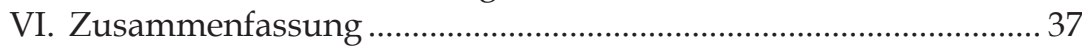

Kapitel 2: Problem: Unmittelbarkeitsprinzip im Verbriefungsrecht

§ 3 Die "dingliche Komponente“ bei Verbriefungstransaktionen ....... 41

I. Vollstreckungsfestigkeit der Vereinbarungstreuhand und das Vormerkungserfordernis nach BGHZ 155, 227............... 43

II. Auswirkungen von BGHZ 155, 227 auf die true sale

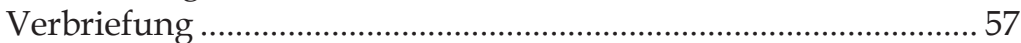

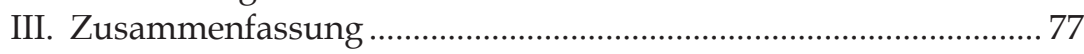

Kapitel 3: Lösung: Schutzwirkungen der Registereintragung

§4 Vollstreckungsrechtlicher Registerschutz

I. Aussonderungsrecht kraft Eintragung,

$\S 22 \mathrm{j}$ Abs. 1 Satz 1 KWG 
II. Aussonderungsanspruch an Surrogaten,

$\S 22 \mathrm{j}$ Abs. 1 Satz 2 KWG

III. Drittwiderspruchsklage kraft Eintragung,

$\S 22 \mathrm{j}$ Abs. 1 Satz 3 KWG.

§ 5 Kein Verfügungsschutz kraft Eintragung ....................................... 171

I. Wirksamkeit nachträglicher Verfügungen ................................. 172

II. Grundsatz: Unwirksamkeit rechtsgeschäftlicher

Verfügungsverbote, § 137 BGB.

III. Verfügungsschutz de lege ferenda .............................................. 184

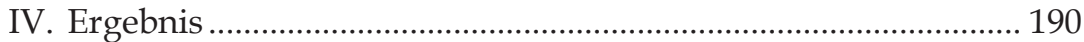

§ 6 Einwendungsschutz der Schuldner und Sicherheitenbesteller.... 193

I. Systematisierung nach Schutzadressaten,

$\S 22 \mathrm{j}$ Abs. 2 KWG

II. Einwendungsschutz der Schuldner,

$\S 22 \mathrm{j}$ Abs. 2 Satz 1-2 KWG

III. Einwendungsschutz der Sicherheitenbesteller,

$\S 22 \mathrm{j}$ Abs. 1 Satz 1-5 KWG.

IV. Zusammenfassung.

$\S 7$ Schutz gegen beeinträchtigende Maßnahmen des

Refinanzierungsunternehmens § 22j Abs. 3 KWG

I. Schutz der Übertragungsansprüche,

$\S 22 \mathrm{j}$ Abs. 3 Satz 1 KWG

II. Anfechtungsschutz der Drittgläubiger,

$\S 22 \mathrm{j}$ Abs. 3 Satz 2 KWG.

\section{Kapitel 4: Schluss}

§ 8 Ergebnisse 223

I. Wesentliche Ergebnisse 223

II. Ergebnisse zur Entstehungsgeschichte der $\S \S 22 \mathrm{a}-22 \mathrm{o} \mathrm{KWG}$ 224

III. Ergebnisse zu der Problemstellung durch BGHZ 155, 227......... 225

IV. Ergebnisse zu den gesetzlichen Wirkungen der Registereintragung 226 


\section{Inhaltsverzeichnis}

Vorwort................................................................................................. VII

Inhaltsübersicht ......................................................................................... IX

\section{Kapitel 1: \\ Einführung}

§ 1 Einleitung ......................................................................................................... 3

I. Gegenstand der Untersuchung.......................................................... 3

II. Anlass der Untersuchung................................................................... 5

1. Konzentration auf die Rechtsfolgen der

Registereintragung 5

2. Besiegelt das Refinanzierungsregister den Streit zum Unmittelbarkeitsprinzip?.......................................................... 6

III. Themeneingrenzung und Gang der Untersuchung .......................... 7

§ 2 Entstehungsgeschichte der Vorschriften §§ 22a-22o KWG ................. 9

I. Gesetzgeberische Bemühungen zur Förderung des

ABS-Marktes

1. Konkurrenzfähigkeit auf den europäischen

Verbriefungsmärkten...

a) Erste Anzeichen einer Erholung an den europäischen Verbriefungsmärkten nach der Finanzkrise 2008/2009?

b) Nachholbedarf des deutschen Verbriefungsmarktes auch nach der Finanzkrise.

2. Zögerliche Adaption von true sale in Deutschland in seinen Anfängen ...................................................................... 13

3. Die Bifurkation des deutschen Verbriefungsmarktes..............15

a) Konkurrent oder Komplementär zum deutschen Pfandbrief? ................................................................... 16

b) Förderung durch die True Sale Initiative ............................ 17

4. Vorreiterrolle Großbritanniens für den europäischen true sale 
II. Politischer Vorprozess zur Schaffung eines

Verbriefungsregisters

1. Finanzmarktförderplan (2003) ................................................. 19

2. True sale und das BCG-Gutachten (2004) ................................ 21

a) Insolvenzrechtliche Anerkennung der

Vereinbarungstreuhand beim true sale ............................ 21

b) "Verbriefungsregister" als Lösung der Treuhandfrage... 22

3. Pfandbriefe und die HypoBG-Novelle (2004) ......................... 22

a) Insolvenzrechtliche Anerkennung der

Vereinbarungstreuhand beim Pfandbrief ......................... 23

b) Erlaß des $\S 1$ Abs. 2 PfandBG in Erwartung einer gesetzlichen Lösung der Treuhandfrage

III. Referentenentwurf zur Schaffung des

Refinanzierungsregisters

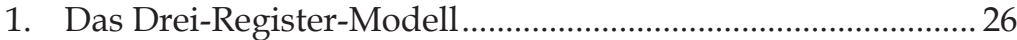

2. Die Übertragungsfiktion des § 22i KWG-E ............................. 27

IV. Gesetz zur Schaffung eines Refinanzierungsregisters .................. 28

1. Die neu eingefügten Regelungen in $\S \S 22 a-22 o$ KWG............ 28

2. Annexcharakter des Gesetzes unterstreicht die Dringlichkeit der Regelungen.................................................29

3. Neue Begriffsbestimmungen der § 1 Abs. 24-26 KWG .......... 29

4. Führung und Kontrolle des Refinanzierungsregisters ............ 31

a) Privat geführtes Registerverfahren ................................... 31

b) Grundsatz der Eigen-Registerführung durch ein Kreditinstitut

c) Formelle Überprüfung der Registerführung durch den Verwalter

d) Sachwalter als „back-up servicer" ...................................... 33

V. Gesetz zur Fortentwicklung des Pfandbriefrechts ........................ 34

1. Erweiterung des Vollstreckungsschutzes,

$\S 22 \mathrm{j}$ Abs.1 Satz 3 KWG

2. Erweiterungen der Legaldefinition

Refinanzierungsunternehmen, § 1 Abs. 24 HS. 1 KWG

3. Kreditinstitute mit Sitz im europäischen Wirtschaftsraum, § 22a Abs. 1 Var. 3 KWG........................................................... 35

4. Erleichterung der Konsortialfinanzierung ............................... 36

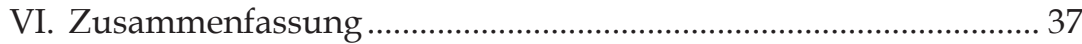




\section{Kapitel 2:}

\section{Problem: Unmittelbarkeitsprinzip im Verbriefungsrecht}

§ 3 Die "dingliche Komponente " bei Verbriefungstransaktionen ....... 41

I. Vollstreckungsfestigkeit der Vereinbarungstreuhand und das Vormerkungserfordernis nach BGHZ 155, 227.

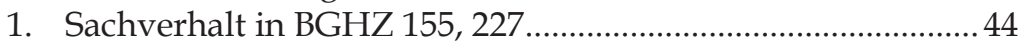

2. Rechtliche Wertungen von BGHZ 155, 227 .............................. 44

a) Die „Zwei Komponenten" der echten Treuhand .............. 44

b) Irrelevanz der Treuhand bei der Aussonderung im Liegenschaftsrecht........................................................... 55

II. Auswirkungen von BGHZ 155, 227 auf die true sale

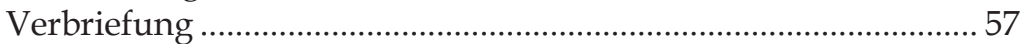

1. Treuhandverhältnisse bei true sale-Transaktionen..................57

a) Erwerbsvorgang als Wesensmerkmal von true sale ........ 57

b) Problem: Übertragung von Sicherheiten ........................... 58

c) Lösung: Treuhandmodell in der Verbriefungspraxis ...... 64

2. Wirkungslosigkeit des Treuhandmodells im Lichte von BGHZ 155, 227.

a) Fehlende „dingliche Komponente“ des Treuhandmodells bei ABS

b) Grundbuchrecht als anachronistisches Hindernis moderner Finanzinstrumente

3. Sonderproblem: die Doppeltreuhand bei der ABSVerbriefung. 71

a) Haftungsrechtliche Zuordnung des Treuguts .................. 72

b) Besondere Verbindung der Sicherungstreuhand zur gesicherten Forderung 74

c) Aussonderungsrecht des Sicherungstreugebers............... 75

III. Zusammenfassung 


\section{Kapitel 3: \\ Lösung: Schutzwirkungen der Registereintragung}

§4 Vollstreckungsrechtlicher Registerschutz

I. Aussonderungsrecht kraft Eintragung,

$\S 22 \mathrm{j}$ Abs. 1 Satz 1 KWG

1. Gesetzliche Regelung des § 22j Abs. 1 Satz 1 KWG $\mathrm{iVm} \S 47 \mathrm{InsO}$ 82

a) Gegenstände des Refinanzierungsunternehmens............. 82

b) Ordnungsgemäße Eintragung ............................................. 93

c) Insolvenz des Refinanzierungsunternehmens.................... 94

d) Anspruch auf Übertragung des Berechtigten .................... 98

e) Inhalt des Aussonderungsanspruchs..................................98

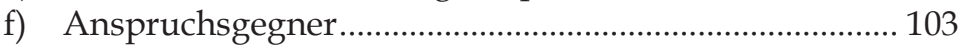

2. Grundgedanke: Erhalt der Deckungsmasse ............................ 104

a) Besondere Verbindung zwischen Anleger und Deckungswerte.................................................................. 104

b) Herstellung der Verbindung durch die Haftungsmasse

3. Rechtliche Ausgestaltung des Vollstreckungsschutzes.........116

a) Adaption des treuhänderischen Grundkonzepts aus § 392 Abs. 2 HGB ...................................................... 117

b) Der Kompromiss des \& 22i KWG-E .................................... 124

c) Der Minimalismus der Rechtsfolgenverweisung in $\S 22 j \mathrm{KWG}$

d) Zurück zur Fiktionswirkung entsprechend $\S 392$ Abs. 2 HGB?

e) Zwischenergebnis ............................................................... 139

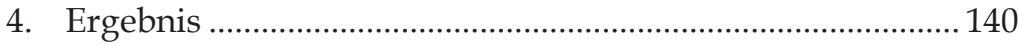

II. Aussonderungsanspruch an Surrogaten,

\& 22j Abs. 1 Satz 2 KWG.

1. Gesetzliche Regelung des § 22j Abs. 1 Satz 2 KWG ............... 141

a) „an die Stelle“ getretene Gegenstände............................... 142

b) Rechtsfolge der Aussonderung von Surrogaten.............. 143

2. Grundgedanke: Wertmäßiger Erhalt der Deckungsmasse.. 143

3. Realisierung durch gesonderten

Aussonderungsanspruch des Übertragungsberechtigten ... 145 
a) Folge der Ablehnung an das

Unmittelbarkeitsprinzips ................................................... 145

b) Kein Ersatzaussonderungsrecht iSd § 48 InsO ............... 147

c) Sonderproblem: Vermengungsgefahr (commingling risk)

4. Ergebnis 151

III. Drittwiderspruchsklage kraft Eintragung,

$\S 22 \mathrm{j}$ Abs. 1 Satz 3 KWG

1. Gesetzliche Regelung des § 22j Abs. 1 Satz 3 KWG iVm § $771 \mathrm{ZPO}$

a) Tatbestand: Zwangsvollstreckung und

Arrestvollziehung in Forderungen und Sicherheiten.... 154

b) Rechtsfolgenverweisung auf § 771 ZPO . 154

2. Grundgedanke: Schutz einzelner Deckungswerte vor der Einzelvollstreckung 155

a) Gefährdung der Deckungsmasse in der Einzelvollstreckung/ Arrestvollziehung 155

b) Gleicher Schutzgedanke bei § 29 PfandBG 156

c) Zwischenergebnis 157

3. Rechtliche Ausgestaltung des Einzelvollstreckungsschutzes kraft Eintragung 157

a) Gesetzeslage vor 26.3.2009 - Inkrafttreten des Gesetzes zur Fortentwicklung des Pfandbriefrechts...... 160

b) Gesetzeslage nach 26.3.2009 - Inkrafttreten des Gesetzes zur Fortentwicklung des Pfandbriefrechts...... 168

4. Ergebnis 170

§ 5 Kein Verfügungsschutz kraft Eintragung

I. Wirksamkeit nachträglicher Verfügungen 172

1. Gesetzeslage vor dem 26.3.2009 - Vor Inkrafttreten des Gesetzes zur Fortentwicklung des Pfandbriefrechts ..... 173

2. Gesetzeslage nach dem 26.3.2009 - Nach Inkrafttreten des Gesetzes zur Fortentwicklung des Pfandbriefrechts ..... 174

3. Unwirksamkeit der Verfügungen während der Schwebezeit, § 161 BGB 176

4. Zwischenergebnis 
II. Grundsatz: Unwirksamkeit rechtsgeschäftlicher Verfügungsverbote, § 137 BGB

1. Verfügungsbeschränkung aufgrund haftungsmäßiger Zuweisung in § 22j KWG?

a) Bejahung des Verfügungsschutzes bei der Kommission, § 392 Abs. 2 HGB

b) Ablehnung des Vollstreckungsschutzes bei der Treuhand

c) Übertragbarkeit der Argumente auf das Refinanzierungsregister?

d) Zwischenergebnis

2. Das Problem: Nichtvereinbarkeit mit dem allgemeinen Gesetzeszweck

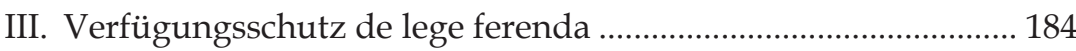

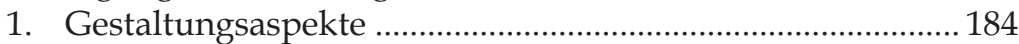

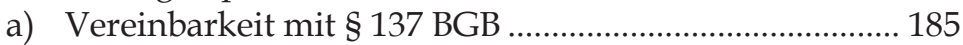

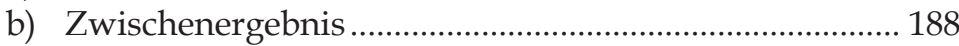

2. Verknüpfung von Vollstreckungsschutz und

Verfügungsschutz bei § 22j KWG.

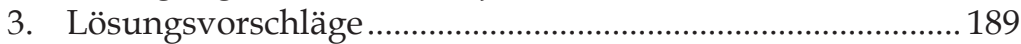

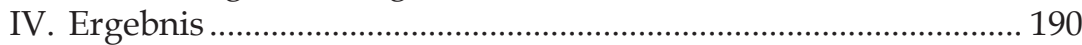

§ 6 Einwendungsschutz der Schuldner und Sicherheitenbesteller..... 193

I. Systematisierung nach Schutzadressaten, § 22j Abs. 2 KWG..... 195

1. Schutz der Schuldner, § 22j Abs. 2 Satz 1-2 KWG ................. 195

2. Schutz der Sicherheitenbesteller,

$\S 22$ j Abs. 2 Satz 1-5 KWG

3. Replik zur Bankgeheimnis-Rechtsprechung,

$\S 22 \mathrm{j}$ Abs. 2 Satz 6 KWG

II. Einwendungsschutz der Schuldner,

$\S 22 \mathrm{j}$ Abs. 2 Satz 1-2 KWG.

1. Einwendungen und Einreden vor Rechtsübergang, Satz 1

a) Tatbestand.

b) Rechtsfolge: Uneingeschränkte Geltendmachung der Einwendungen

c) Grundgedanke: Keine Veränderung der sachenrechtlichen Zuordnung 
d) Lösungsvorschlag: Entsprechende Anwendung des $\S 394$ BGB

2. Einwendungen der Schuldner nach Rechtsübergang, Satz 2 204

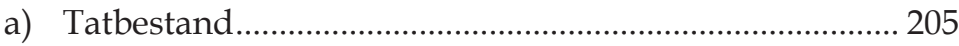

b) Rechtsfolge: Verweis auf die allgemeinen Abtretungsregeln, §§ 404, 406-410 BGB.......................... 206

c) Rechtsgedanke: Schuldnerschutz nach $\S \S 404,406-410$ BGB 207

d) Sonderregelungen im Zeitpunkt nach Aussonderung und vor Übertragung?

III. Einwendungsschutz der Sicherheitenbesteller, § 22j Abs. 1 Satz 1-5 KWG 209

1. Einwendungsschutz vor Rechtsübergang, Satz 1 ................. 209

2. Einwendungsschutz nach Rechtsübergang, Satz 2 ............... 210

3. Einwendungsschutz nach Zweiterwerb von Hypothek und Grundschuld, Satz 3

4. Ausschluss des gutgläubigen einredefreien Zweiterwerbs, Satz 4 und 5

IV. Zusammenfassung

\section{$\S 7$ Schutz gegen beeinträchtigende Maßnahmen des}

Refinanzierungsunternehmens § 22j Abs. 3 KWG

I. Schutz der Übertragungsansprüche, § 22j Abs. 3 Satz 1 KWG .. 215

1. Regelungsgehalt: Schutz der Übertragungsansprüche

2. Grundgedanke: Der Anwartschaftsähnliche Schutz der Registereintragung

II. Anfechtungsschutz der Drittgläubiger,

§ 22j Abs. 3 Satz 2 KWG.

1. Regelungsgehalt: Gläubigerschutz vor missbräuchlicher Verkürzung der Insolvenzmasse

2. Grundgedanke: Keine Instrumentalisierung des Registers zu unlauteren Vermögensverschiebungen 


\section{Kapitel 4: \\ Schluss}

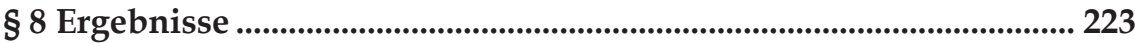

I. Wesentliche Ergebnisse ................................................................ 223

II. Ergebnisse zur Entstehungsgeschichte der $\S \S 22 a$ - 22o KWG ..................................................................... 224

III. Ergebnisse zu der Problemstellung durch BGHZ 155, 227......... 225

IV. Ergebnisse zu den gesetzlichen Wirkungen der

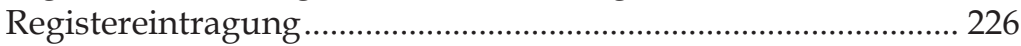

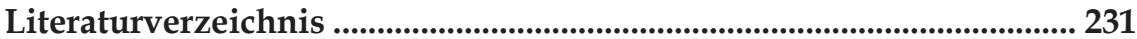

